\title{
Bistability of squeezing and entanglement in cavity magnonics
}

\author{
Zhi-Bo Yang, ${ }^{1}$ Hua Jin, ${ }^{1}$ Jing-Wen Jin, ${ }^{1}$ Jian-Yu Liu, ${ }^{1}$ Hong-Yu Liu, ${ }^{1, *}$ and Rong-Can Yang ${ }^{2,3,+}$ \\ ${ }^{1}$ College of Science, Yanbian University, Yanji, Jilin 133002, China \\ ${ }^{2}$ Fujian Provincial Key Laboratory of Quantum Manipulation \\ and New Energy Materials and College of Physics and Energy, \\ Fujian Normal University, Fuzhou 350117, China \\ ${ }^{3}$ Fujian Provincial Collaborative Innovation Center for Optoelectronic \\ Semiconductors and Efficient Devices, Xiamen 361005, China
}

(Received 30 October 2020; accepted 19 April 2021; published 18 May 2021)

\begin{abstract}
We demonstrate how magnon Kerr nonlinearity creates bistability of quantum states when the magnon mode is strongly driven by a microwave field in a nonlinear cavity magnonic system. Numerical simulation results with experimentally feasible parameters show that the method where the system is driven far from equilibrium is a reliable way to achieve squeezed states for magnons and photons and to carry out magnon-photon entanglement, revealing mysterious phenomena of bistability. In addition, the Kittel mode can jump from one state to another one near two switching points, thus achieving the hysteresis loop phenomenon. Our results indicate that bistable quantum states could provide a way to study macroscopic quantum bistable phenomena in nonlinear systems and also can be found in broad applications in magnetic spintronics.
\end{abstract}

DOI: 10.1103/PhysRevResearch.3.023126

\section{INTRODUCTION}

Hybrid quantum systems consisting of different subsystems have recently drawn considerable attention [1,2] because of the fundamental importance and promising applications in quantum information processing. Among them, cavity magnonics [3-14] seems to be a suitable candidate for the study of multiple quantum correlation because magnons have distinguishing advantages of a long lifetime, easy tunability, etc. Several cavity magnonic systems (CMSs) were theoretically proposed [15-17] and experimentally demonstrated [12,18-22]. It was found that strong coupling between the microwave cavity mode and the Kittel mode [23] (a spatially uniform mode of the ferrimagnetic spin waves) in a small yttrium iron garnet (YIG) sample with a low damping rate can be achieved [12,18-21], which is a challenging task for spin ensembles in paramagnetic materials. This CMS has many exotic phenomena, such as cavity magnon polaritons [18-20,24,25], the magnon Kerr effect [26-28], nonclassical states [3-5,13], magnoninduced transparency [29], optical diodes [6], bidirectional microwave-optical conversion [30], magnon dark modes [31], cooperative polariton dynamics [32], and non-Hermitian physics $[29,33,34]$, etc. Moreover, magnons can also inter-

\footnotetext{
*liuhongyu@ybu.edu.cn

†rcyang@fjnu.edu.cn
}

Published by the American Physical Society under the terms of the Creative Commons Attribution 4.0 International license. Further distribution of this work must maintain attribution to the author(s) and the published article's title, journal citation, and DOI. act with phonons directly [35] or superconducting qubits indirectly $[36,37]$ to form different hybrid systems. In particular, magnons can be coupled to visible/near-infrared light through the magneto-optical effect (i.e., cavity optomagnonics) [38-45] and to microwave photons through magnetic dipole interaction (i.e., cavity magnonics) [3-14,44].

For a CMS, in addition to hybridization between magnons and microwave photons, the nonlinear effect can also play an important role. From a theoretical and experimental point of view, this nonlinearity, originating from magnetocrystalline anisotropy in a YIG sample [46,47], is related to the magnon self-nonlinear Kerr effect. So far, the nonlinearity-induced frequency shift [14,27], entangled state [7], nonreciprocal transmission [6], bistability of cavity magnon polaritons [26], and magnon-induced high-order sideband [28] have been demonstrated with the method. However, as far as we know, bistability of quantum states based on the magnon selfnonlinear Kerr effect has not yet been demonstrated. Thus, in this research, we show the bistability of squeezed states and entangled states in a nonlinear CMS. For a wide range of parameters, the squeezing degree of magnons and magnonphoton entanglement are found to jump up (or down) sharply at switching points where quantum states of the magnon mode transit from one state to another. We show that the magnon self-nonlinear Kerr effect is a reliable way to realize squeezed magnons and magnon-photon entanglement by driving the system far from equilibrium. We also demonstrate the effect of magnon-photon state-swap interaction on squeezing and entanglement and the strong robustness of quantum states against the cavity damping rate and environment temperature.

As an important nonlinear phenomenon in physics, bistability is not only of fundamental interest in studying dissipative quantum systems [48,49] but also has potential 


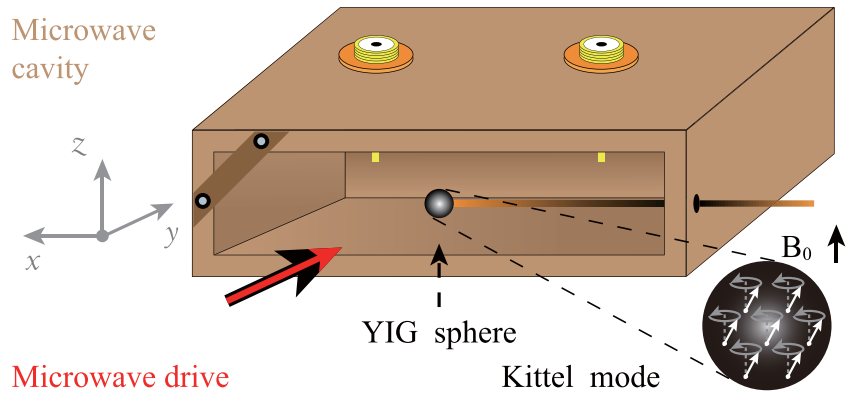

FIG. 1. Sketch of a YIG sphere coupled to a microwave cavity. The YIG sphere, which is magnetized to saturation by a bias magnetic field $\mathbf{B}_{0}$ aligned along the $z$ direction, is mounted near the cavity wall, where the magnetic field $\mathbf{B}$ of the cavity mode $\left(\mathrm{TE}_{110}\right)$ is the strongest and polarized along the $x$ direction to excite the magnon mode in the YIG sphere. The magnon mode is driven by a microwave field along the $y$ direction.

applications in switches [50,51] and memories [52,53]. However, as two special types of quantum states, squeezed and entangled states can be used not only to improve measurement sensitivity [54] but also to study decoherence at large scales [55]. In addition, squeezed and entangled states are vital ingredients for continuous-variable information processing [56]. Our scheme offers a method for exploring these applications and shows that the bistability of quantum states can serve as a bridge or transducer between these applications, which paves the way for using one effect to induce or control the other.

The research is organized as follows. In Sec. II, we introduce the fundamental model and equation of motion of our proposal. In Sec. III, we demonstrate the classical bistability of the CMS by solving the third-order nonlinear equation. In Secs. IV and V, we study the bistability of the squeezed state and the entangled state induced by classical bistability, respectively. In Sec. VI, we explain the resulting bistable squeezed (entangled) state from the perspective of principle and mechanism. Then we analyze the parameter feasibility of the CMS from the experimental point of view in Sec. VII. Conclusions and remarks are given in Sec. VIII.

\section{MODEL AND EQUATION OF MOTION}

As schematically shown in Fig. 1, we study the Kittel mode [23] in a small YIG sphere which is strongly coupled to a three-dimensional (3D) copper cavity. At the same time, the Kittel mode is driven by a microwave field. The Hamiltonian for the whole system reads

$$
\begin{aligned}
\mathcal{H}= & \frac{1}{2} \int\left(\varepsilon_{0} \mathbf{E}^{2}+\frac{\mathbf{B}^{2}}{\mu_{0}}\right) d \tau-\int \mathbf{M} \cdot \mathbf{B}_{0} d \tau-\mu_{0} \int \mathbf{M} \cdot \mathbf{B} d \tau \\
& -\frac{\mu_{0}}{2} \int \mathbf{M} \cdot \mathbf{H}_{\mathrm{an}} d \tau-\mu_{0} \int \mathbf{M} \cdot \mathbf{H}_{\mathrm{d}} d \tau,
\end{aligned}
$$

where $\mathbf{B}_{0}=B_{0} \mathbf{e}_{z}$ is an applied static magnetic field in the $z$ direction in order for the YIG sphere to be magnetized and $\mathbf{e}_{i=x, y, z}$ denotes the unit vector in the rectangular coordinate system (see Fig. 1). $\mathbf{E}$ and $\mathbf{B}$ are, respectively, the electric and magnetic components of the electromagnetic wave inside the cavity, and $\varepsilon_{0}\left(\mu_{0}\right)$ is the vacuum permittivity (permeability). $\mathbf{M}=\hbar \gamma \mathbf{S} / V_{m} \equiv\left(M_{x}, M_{y}, M_{z}\right)$ is the magnetization of the YIG sphere, with $\gamma$ being the gyromagnetic ratio. $V_{m}$ is the volume of the YIG sphere. $\mathbf{S} \equiv\left(S_{x}, S_{y}, S_{z}\right)$ stands for the collective spin operator. The magnetic field $\mathbf{B}$ of the cavity mode is polarized along the $x$ direction, and $\mathbf{B}=$ $-\sqrt{\hbar \omega_{a} /\left(\mu_{0} V_{a}\right)}\left(a^{\dagger}+a\right) \mathbf{e}_{x}$, where $a$ and $a^{\dagger}$ are the annihilation and creation operators of the cavity mode at the frequency $\omega_{a} . V_{a}$ is the volume of the cavity. $\mathbf{H}_{\mathrm{an}}=-\left(2 K_{a n} M_{z} / M^{2}\right) \mathbf{e}_{z}$ is the anisotropic field due to the magnetocrystalline anisotropy and has only a $z$ component owing to the 100 crystallographic axis being aligned along the applied static magnetic field [14] $K_{a n}$ is the dominant first-order anisotropy constant, and $M$ is the saturation magnetization. The Holstein-Primakoff transform yields [57] $S_{z}=S-m^{\dagger} m, S_{+}=m \sqrt{2 S-m^{\dagger} m}, S_{-}=$ $m^{\dagger} \sqrt{2 S-m^{\dagger} m}$, where $S_{ \pm} \equiv S_{x} \pm i S_{y}$ and $m\left(m^{\dagger}\right)$ represents the magnon annihilation (creation) operator. In this research, a considerable number of magnons is generated by driving microwave field $\mathbf{H}_{\mathrm{d}}=-B_{d} \cos \left(\omega_{d} t\right) \mathbf{e}_{y}$ with frequency $\omega_{d}$ and amplitude $B_{d}$, but low-lying excitations with $\left\langle m^{\dagger} m\right\rangle \ll$ $2 S$ are still satisfied. Therefore, we can safely approximate $S_{+} \simeq \sqrt{2 S} m$ and $S_{-} \simeq \sqrt{2 S} m^{\dagger}$ because $2 S=5 \rho V_{m}$ is a huge number for a micrometer-scale YIG sphere with spin density $\rho=4.22 \times 10^{27} \mathrm{~m}^{-3}[4]$. In this case, one can recast the Hamiltonian (1) into

$$
\begin{aligned}
\mathcal{H} / \hbar= & \omega_{a} a^{\dagger} a-\gamma B_{0} S_{z}+\mu_{0} K_{a n} \gamma^{2} S_{z}^{2} \hbar /\left(M^{2} V_{m}\right) \\
& +g_{s}\left(S_{+}+S_{-}\right)\left(a^{\dagger}+a\right) \\
& +i \Omega_{S}\left(S_{-}-S_{+}\right)\left(e^{i \omega_{d} t}+e^{-i \omega_{d} t}\right),
\end{aligned}
$$

where $g_{s}=\sqrt{\gamma^{2} \mu_{0} \hbar \omega_{a} /\left(4 V_{a}\right)}\left(\Omega_{s}=\mu_{0} \gamma B_{d} / 4\right)$ denotes the coupling strength between each single spin and cavity mode (driving field). Note that the Hamiltonian (2) which consists of counterrotating terms plays an important role in the ultrastrong-coupling regime of light and matter (represented by spins) [58]. However, in our scheme, the total effective Hamiltonian of the CMS under the rotating-wave approximation can be rewritten as

$$
\begin{aligned}
\mathcal{H}_{\mathrm{eff}} / \hbar= & \omega_{a} a^{\dagger} a+\omega_{m} m^{\dagger} m+K m^{\dagger} m m^{\dagger} m \\
& +g\left(m^{\dagger} a+m a^{\dagger}\right)+i \Omega\left(m^{\dagger} e^{-i \omega_{d} t}-m e^{i \omega_{d} t}\right) .
\end{aligned}
$$

It is worth noting that the Hamiltonian (3) is commonly studied in cavity QED [59] and in circuit QED [60]. Here, $\omega_{m}=\gamma B_{0}-2 \mu_{0} K_{a n} \gamma^{2} S \hbar /\left(M^{2} V_{m}\right)$ is the angular frequency of the magnon mode. $K=\mu_{0} K_{a n} \gamma^{2} \hbar /\left(M^{2} V_{m}\right)$ represents the Kerr nonlinear coefficient, and $g=\sqrt{2 S} g_{s}$ is the magnonphoton coupling rate. $\Omega=\sqrt{2 S} \Omega_{s}$ is Rabi frequency [3-5]. The dissipative dynamics of the CMS is described by a set of quantum Langevin equations (QLEs) [61]:

$$
\begin{aligned}
\dot{a} & =-\left(i \Delta_{a}+\kappa_{a}\right) a-i g m+\sqrt{2 \kappa_{a}} a^{\text {in }}, \\
\dot{m} & =-\left(i \Delta_{m}+\kappa_{m}\right) m-i g a+\sqrt{2 \kappa_{m}} m^{\text {in }}-2 i K m^{\dagger} m m+\Omega,
\end{aligned}
$$

where $\Delta_{o=a, m}=\omega_{o}-\omega_{d} . \kappa_{a}$ and $\kappa_{m}\left(a^{\text {in }}\right.$ and $\left.m^{\text {in }}\right)$ represent the damping rates (input noise operators) of the cavity and magnon modes, respectively. The input noise operators have zero mean and are characterized by the following correlation 
functions:

$$
\begin{aligned}
& \left\langle o^{\mathrm{in} \dagger}(t) o^{\mathrm{in}}\left(t^{\prime}\right)\right\rangle=n_{o} \delta\left(t-t^{\prime}\right), \\
& \left\langle o^{\mathrm{in}}(t) o^{\mathrm{in} \dagger}\left(t^{\prime}\right)\right\rangle=\left(n_{o}+1\right) \delta\left(t-t^{\prime}\right),
\end{aligned}
$$

with $n_{o=a, m}=\left\{\exp \left[\hbar \omega_{o} /\left(k_{B} T\right)\right]-1\right\}^{-1}$ being equilibrium mean thermal photon/magnon numbers. $T$ is the environmental temperature, and $k_{B}$ is the Boltzmann constant.

Since the magnon mode is strongly driven by a microwave field, the magnon-photon beam-splitter-like interaction leads to large amplitudes of both the magnon mode and cavity mode, i.e., $|\langle o\rangle| \gg 1$. In this case, one can safely introduce the expansion $o=\langle o\rangle+\delta o$ in the vicinity of steady states by neglecting higher-order fluctuations of the operators. Then, we obtain a set of differential equations for mean values,

$$
\begin{aligned}
\langle\dot{a}\rangle & =-\left(i \Delta_{a}+\kappa_{a}\right)\langle a\rangle-i g\langle m\rangle, \\
\langle\dot{m}\rangle & =-\left(i \Delta_{m}+\kappa_{m}\right)\langle m\rangle-i g\langle a\rangle-2 i K|\langle m\rangle|^{2}\langle m\rangle+\Omega,
\end{aligned}
$$

and linearized QLEs for the quantum fluctuations,

$$
\begin{aligned}
& \delta \dot{a}=-\left(i \Delta_{a}+\kappa_{a}\right) \delta a-i g \delta m+\sqrt{2 \kappa_{a}} a^{\text {in }}, \\
& \delta \dot{m}=-\left(i \tilde{\Delta}_{m}+\kappa_{m}\right) \delta m-i g \delta a+\sqrt{2 \kappa_{m}} m^{\text {in }}+(\mathcal{I}-i \mathcal{R}) \delta m^{\dagger},
\end{aligned}
$$

where $\tilde{\Delta}_{m}=\Delta_{m}+\Delta_{K}$ includes the frequency shift $\Delta_{K}=$ $4 K|\langle m\rangle|^{2}$ caused by the Kerr effect and $\mathcal{R}$ and $\mathcal{I}$ are the real and imaginary parts of $2 K\langle m\rangle^{2}$, respectively. The mean values $\langle a\rangle$ and $\langle m\rangle$ are given by

$$
\begin{aligned}
\langle a\rangle & =-\left[i g /\left(i \Delta_{a}+\kappa_{a}\right)\right]\langle m\rangle, \\
\langle m\rangle & =\Omega /\left[i \Delta_{m}+\kappa_{m}+2 i K|\langle m\rangle|^{2}+g^{2} /\left(i \Delta_{a}+\kappa_{a}\right)\right] .
\end{aligned}
$$

\section{BISTABLE BEHAVIOR INDUCED BY THE KERR EFFECT}

Note that Eq. (8) takes a simpler form,

$$
\langle a\rangle \simeq-\left(g / \Delta_{a}\right)\langle m\rangle, \quad\langle m\rangle \simeq i \Omega /\left(g^{2} / \Delta_{a}-\Delta_{m}-2 K|\langle m\rangle|^{2}\right)
$$

(pure imaginary numbers) when $\left|\Delta_{m}\right|,\left|\Delta_{a}\right| \gg \kappa_{a}, \kappa_{m}$. In this case, $\mathcal{I} \simeq 0$, and $\mathcal{R} \simeq 2 K\langle m\rangle^{2} \simeq-2 K|\langle m\rangle|^{2}$. According to Eq. (8), we have a third-order nonlinear equation for the shifted frequency $\Delta_{K}$,

$$
\Delta_{K}^{3}+t_{2} \Delta_{K}^{2}+t_{1} \Delta_{K}+t_{0}=0
$$

with

$$
\begin{aligned}
t_{2}= & 4 \Delta_{m}+i\left(\xi_{-}-\xi_{+}\right), \\
t_{1}= & 4\left(\Delta_{m}^{2}+\kappa_{m}^{2}\right)+2\left(\kappa_{m}+i \Delta_{m}\right) \xi_{-} \\
& +2\left(\kappa_{m}-i \Delta_{m}\right) \xi_{+}+\xi_{+} \xi_{-}, \\
t_{0}= & -16 K \Omega^{2},
\end{aligned}
$$

where $\xi_{ \pm}=2 g^{2} /\left(\kappa_{a} \pm i \Delta_{a}\right)$ and Eq. (10) implies that $\Delta_{K}$ can show bistable behaviors. In order to show the bistability of the shifted frequency $\Delta_{K}$ induced by the Kerr effect in the steady state when magnons are driven, Fig. 2 describes $\Delta_{K}$ as a function of the magnon frequency $\omega_{m}$ for $\Omega=4 \times 10^{13} \mathrm{~Hz}$ (internal curve) and $\Omega=6 \times 10^{13} \mathrm{~Hz}$ (external curve), where we have employed experimentally

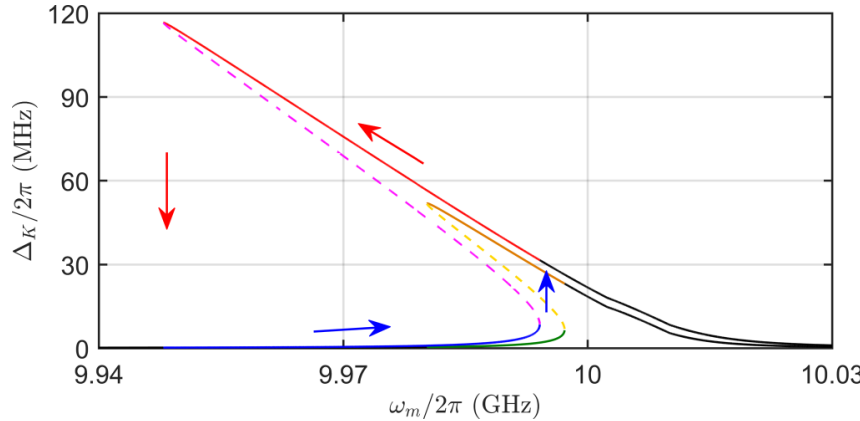

FIG. 2. Shifted frequency $\Delta_{K}$ versus magnon frequency $\omega_{m}$ for $\Omega=4 \times 10^{13} \mathrm{~Hz}$ (internal curve) and $\Omega=6 \times 10^{13} \mathrm{~Hz}$ (external curve). We take $\omega_{a} / 2 \pi=10 \mathrm{GHz}, \kappa_{a} / 2 \pi=\kappa_{m} / 2 \pi=1 \mathrm{MHz}$, $K / 2 \pi=1.56 \mu \mathrm{Hz}, \Delta_{a} / 2 \pi=30 \mathrm{MHz}, g / 2 \pi=33 \mathrm{MHz}$, and $T=$ $20 \mathrm{mK}$.

feasible parameters [7,26,62]: $\omega_{a} / 2 \pi=10 \mathrm{GHz}, \kappa_{a} / 2 \pi=$ $\kappa_{m} / 2 \pi=1 \mathrm{MHz}, g / 2 \pi=33 \mathrm{MHz}, \Delta_{a} / 2 \pi=30 \mathrm{MHz}, T=$ $20 \mathrm{mK}$, and $K / 2 \pi \simeq 1.56 \mu \mathrm{Hz}$ for a $40-\mu \mathrm{m}$-diameter $\mathrm{YIG}$ sphere. In Fig. 2, a hysteresis loop is clearly seen for a wide range of parameters, showing a bistable behavior of $\Delta_{K}$ induced by the Kerr effect in the nonlinear CMS. When the increasing and decreasing directions of $\omega_{m}$ are considered, a counterclockwise hysteresis loop is clearly illustrated, and its area increases with increasing $\Omega$. Thus, we can achieve a higher magnonic response (i.e., a larger frequency shift and effective Kerr coefficient) and a larger bistable region. In order to ensure the validity of low-lying excitation approximation, the condition $\left\langle m^{\dagger} m\right\rangle \ll 2 S$ must be satisfied [4]. For a $40-\mu \mathrm{m}$-diameter $\mathrm{YIG}$ sphere, $2 S \simeq 7 \times 10^{14}$. In Fig. $2, \max \left[\Delta_{K}\right] / 2 \pi \simeq 118 \mathrm{MHz} \rightarrow \max \left[\left\langle m^{\dagger} m\right\rangle\right] \simeq 1.9 \times$ $10^{12} \ll 2 S$, which easily shows our procedure is valid in the parameter regime we considered.

\section{SQUEEZED MAGNON MODE}

\section{A. Quantification of the squeezed magnon mode}

To quantify the quadrature variance of the magnon amplitude and phase, we introduce quadrature operators for the cavity and magnon modes,

$$
\delta x_{o=a, m}=\left(\delta o+\delta o^{\dagger}\right) / \sqrt{2}, \quad \delta y_{o=a, m}=i\left(\delta o^{\dagger}-\delta o\right) / \sqrt{2},
$$

and the quadrature noise operators for the cavity and magnon modes,

$$
\delta x_{o=a, m}^{\text {in }}=\left(o^{\text {in }}+o^{\text {in } \dagger}\right) / \sqrt{2}, \quad \delta y_{o=a, m}^{\text {in }}=i\left(o^{\text {in } \dagger}-o^{\text {in }}\right) / \sqrt{2} .
$$

Then Eq. (7) can be expressed in a more concise form:

$$
\dot{\sigma}(t)=\mathcal{A} \sigma(t)+n(t),
$$

where $\quad \sigma=\left[\delta x_{a}, \delta y_{a}, \delta x_{m}, \delta y_{m}\right]^{T} \quad$ and $\quad n=\left[\sqrt{2 \kappa_{a}} \delta x_{a}^{\text {in }}\right.$, $\left.\sqrt{2 \kappa_{a}} \delta y_{a}^{\text {in }}, \sqrt{2 \kappa_{m}} \delta x_{m}^{\text {in }}, \sqrt{2 \kappa_{m}} \delta y_{m}^{\text {in }}\right]^{T}$ are, respectively, the vectors for quantum fluctuations and noises. In this case, the 
drift matrix $\mathcal{A}$ reads

$$
\mathcal{A}=\left[\begin{array}{cccc}
-\kappa_{a} & \Delta_{a} & 0 & g \\
-\Delta_{a} & -\kappa_{a} & -g & 0 \\
0 & g & \kappa_{+} & \Delta_{+} \\
-g & 0 & \Delta_{-} & \kappa_{-}
\end{array}\right],
$$

where $\Delta_{ \pm}= \pm\left(\Delta_{m}+\Delta_{K}\right)-\mathcal{R}$ and $\kappa_{ \pm}=-\kappa_{m} \pm \mathcal{I}$ (set $\mathcal{I}=$ 0 for simplicity).

Since we are using linearized QLEs, the Gaussian nature of input states will be preserved during the time evolution of the system. The quantum fluctuations are thus the continuous two-mode Gaussian state, which can be completely characterized by a $4 \times 4$ covariance matrix $(\mathrm{CM}) \mathcal{V}$ in the phase space $\mathcal{V}_{i j}\left(t, t^{\prime}\right)=\left\langle\sigma_{i}(t) \sigma_{j}\left(t^{\prime}\right)+\sigma_{j}\left(t^{\prime}\right) \sigma_{i}(t)\right\rangle / 2(i, j=$ $1, \ldots, 4)$. Then the vectors can be obtained straightforwardly by solving the Lyapunov equation,

$$
\mathcal{A} \mathcal{V}+\mathcal{V} \mathcal{A}^{T}=-\mathcal{D}
$$

with $\mathcal{D}=\operatorname{diag}\left[\left(2 n_{a}+1\right) \kappa_{a},\left(2 n_{a}+1\right) \kappa_{a},\left(2 n_{m}+1\right) \kappa_{m}\right.$, $\left.\left(2 n_{m}+1\right) \kappa_{m}\right]$ defined through $\mathcal{D}_{i j} \delta\left(t-t^{\prime}\right)=\left\langle n_{i}(t) n_{j}\left(t^{\prime}\right)+\right.$ $\left.n_{j}\left(t^{\prime}\right) n_{i}(t)\right\rangle / 2$. Obviously, the last two diagonal elements, $\mathcal{V}_{33}$ and $\mathcal{V}_{44}$, of $\mathcal{V}$ represent variances for the magnon amplitude and phase, respectively. Note that self-interaction between magnons occurs because of Kerr nonlinearity, which makes the drift matrix $\mathcal{A}$ different from that in the case where only magnon-photon beam-splitter-like interaction is involved. Therefore, the stability of the system is the first prerequisite for the effectiveness of our scheme. According to the Routh-Hurwitz criterion [63], the system is stable and reaches its steady state when all eigenvalues of the matrix $\mathcal{A}$ have negative real parts. Therefore, we start our analysis by determining eigenvalues of the matrix $\mathcal{A}$ (i.e., $|\mathcal{A}-\lambda \mathbf{I}|=0$ ) and make sure the stability conditions are all satisfied in the following sections.

\section{B. Bistable behavior of the squeezed magnon mode and Wigner function}

We first show that the magnon mode can be squeezed by driving the system far from equilibrium. Figure 3(a) illustrates the quadrature variance of the magnon phase $\left\langle\delta y_{m}^{2}\right\rangle$ as a function of the magnon frequency $\omega_{m}$. When considering the increasing and decreasing directions of $\omega_{m}$, the quadrature variance of the magnons shows a bistable behavior similar to that shown in Fig. 2. When $\omega_{m} / 2 \pi=9.95 \mathrm{GHz}$, the quadrature variance of the lower stable branch is about 0.5 , and the quadrature variance of the upper stable branch is about 0.37 , as indicated by the blue and red lines in Fig. 3(a), respectively. For a wide range of parameters, the squeezing degree of the magnon mode is found to jump up or down sharply at the switching points where the quantum state of the magnon transitions from one state to another (e.g., high-squeezing state to low-squeezing state or coherent state). Next, in order to demonstrate the bistable behavior of the squeezed magnon mode to be more intuitively in phase space, we show the quasiprobability distribution function in phase space in Figs. 3(b) and 3(c) when $\omega_{m} / 2 \pi=9.95 \mathrm{GHz}$ based on the parameter mechanism of the red and blue lines in Fig. 3(a), respectively. To this end, it is necessary to introduce the Wigner function. Due to the above linearized system dynamics and
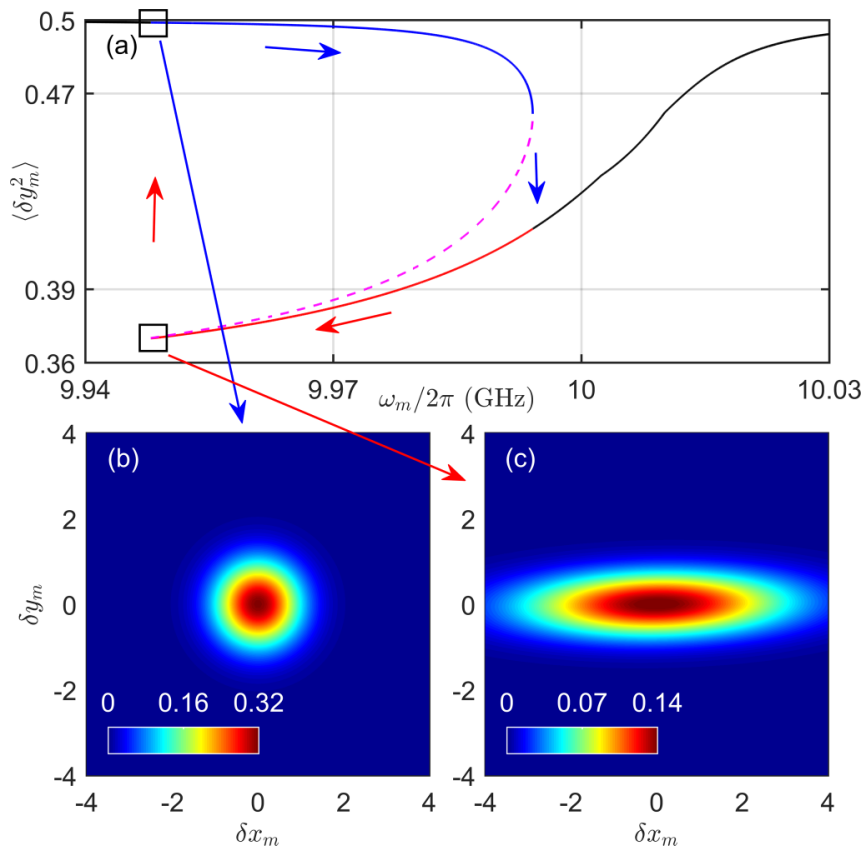

FIG. 3. (a) Variance of the magnon phase quadrature $\left\langle\delta y_{m}^{2}\right\rangle$ versus magnon frequency $\omega_{m}$, where $\Omega=6 \times 10^{13} \mathrm{~Hz}$ and $\Delta_{K}=-2 \mathcal{R}$. Wigner functions of the magnon mode in cases (b) with the bistable region parameters of the blue line and (c) with the bistable region parameter of the red line when $\omega_{m} / 2 \pi=9.95 \mathrm{GHz}$. The other parameters are the same as in Fig. 2.

the zero-mean Gaussian nature of the quantum noises, the quantum steady state of the system is a Gaussian state [64,65]. Hence, as long as CM is obtained, the Wigner function of the magnon mode can be expressed as $[66,67]$

$$
\mathcal{W}\left(\sigma_{m}\right)=\frac{1}{2 \pi \sqrt{\operatorname{det}\left[\mathcal{V}_{m}\right]}} \exp \left[-\frac{1}{2} \sigma_{m}^{T} \mathcal{V}_{m}^{-1} \sigma_{m}\right],
$$

where $\sigma_{m}=\left[\delta x_{m}, \delta y_{m}\right]^{T}$ refers to a two-dimensional vector and $\mathcal{V}_{m}$ is the CM for the magnon mode. In Figs. 3(b) and $3(\mathrm{c})$, Wigner functions of the magnon mode with the bistable parameter region of blue line and red line, respectively, are demonstrated. One can also observe in Fig. 3(b) that the quantum state of the magnon mode is a coherent state, where the magnon mode possesses vacuum fluctuations. On the contrary, Fig. 3(c) illustrates that the quantum state of the magnon mode for the red line is a squeezed state, where the magnon mode is squeezed as a result of the systems far from equilibrium and $\left\langle\delta y_{m}^{2}\right\rangle<\left\langle\delta y_{m}^{2}\right\rangle_{\text {vac }}$, with $\left\langle\delta y_{m}^{2}\right\rangle_{\text {vac }}=0.5$, denotes vacuum fluctuations.

\section{Magnon-photon state-swap interaction}

The magnon squeezed state due to the magnon selfnonlinear Kerr effect is further demonstrated in Fig. 4 when the CMS does not have a linear relation of $\Delta_{K}=-2 \mathcal{R}$, where the squeezing degree of the magnon mode is expressed in decibels by $-10 \log _{10}\left[\left\langle\delta y_{m}^{2}\right\rangle /\left\langle\delta y_{m}^{2}\right\rangle_{\text {vac }}\right]$. Figure 4 shows the features of $\left\langle\delta y_{m}^{2}\right\rangle$ (top row) and $\left\langle\delta y_{a}^{2}\right\rangle$ (bottom row) as functions of some key parameters in the system [68]. First of all, we observe from Figs. 4(a) and 4(d) that Kerr nonlinearity is responsible for creating squeezed states of the magnon and 

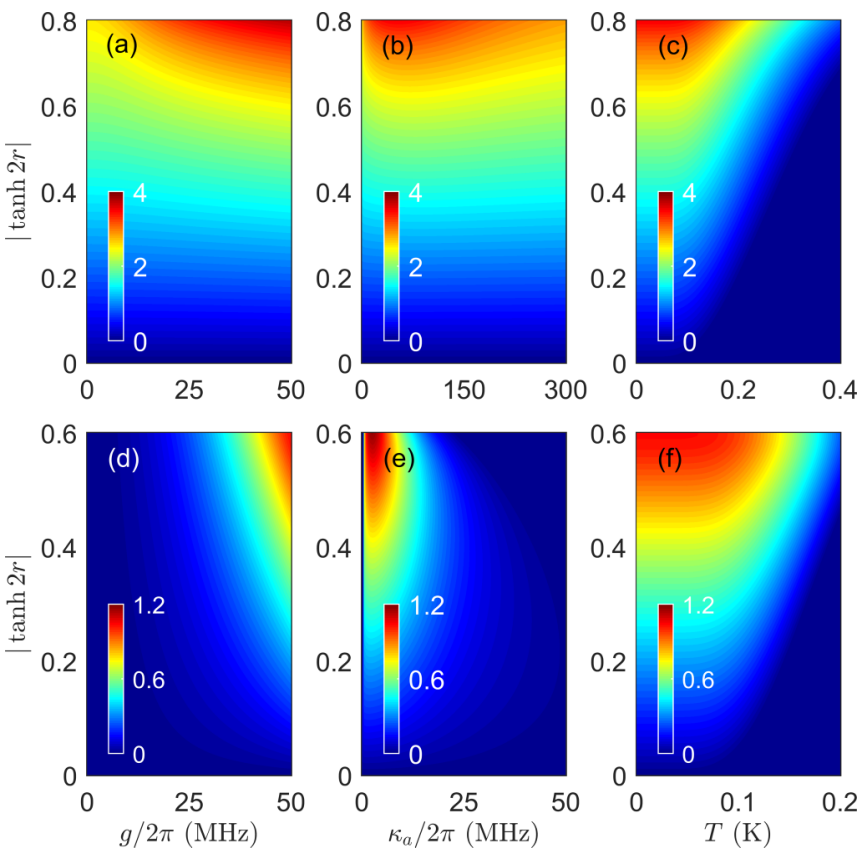

FIG. 4. Three-dimensional plots of quadrature variance for (a)(c) the magnon phase $\left\langle\delta y_{m}^{2}\right\rangle$ and (d)-(f) the cavity phase $\left\langle\delta y_{a}^{2}\right\rangle$ (in $\mathrm{dB}$ ). We set $\kappa_{a} / 2 \pi=50 \mathrm{MHz}, T=20 \mathrm{mK}$ in (a), $g / 2 \pi=33 \mathrm{MHz}, T=$ $20 \mathrm{mK}$ in (b), $g / 2 \pi=33 \mathrm{MHz}, \kappa_{a} / 2 \pi=50 \mathrm{MHz}$ in (c), $\kappa_{a} / 2 \pi=$ $5 \mathrm{MHz}, T=20 \mathrm{mK}$ in (d), $g / 2 \pi=50 \mathrm{MHz}, T=20 \mathrm{mK}$ in (e), and $g / 2 \pi=50 \mathrm{MHz}, \kappa_{a} / 2 \pi=5 \mathrm{MHz}$ in (f) and $\Delta_{K} / 2 \pi=280 \mathrm{MHz}$, $\omega_{m} / 2 \pi=9.95 \mathrm{GHz}$ for all plots. The other parameters are the same as in Fig. 2.

cavity modes, which is evident from the fact that the squeezing degree dies out when $|\tanh 2 r|=0$, where $|\tanh 2 r|=$ $\left|\mathcal{R} /\left(\Delta_{m}+\Delta_{K}\right)\right|$ is the squeezed parameter of the CMS (we will verify that later; see Sec. VI). In addition, squeezing of the cavity mode is derived from the magnon-photon beam-splitter-like (state-swap) interaction, evident from the fact that the squeezing degree of the cavity mode dies out when $g / 2 \pi=0$. As the coupling rate increases, the magnon squeezing degree decreases [69], while the cavity squeezing increases, implying that the squeezing has been partially transferred from the magnons to the cavity. For example, for $|\tanh 2 r|=0.6$ the cavity squeezing is $0 \mathrm{~dB}$ when $g / 2 \pi=0$, and the cavity squeezing is about $1 \mathrm{~dB}$ when $g / 2 \pi=50 \mathrm{MHz}$. In other words, magnons and photons are squeezed simultaneously. This is consistent with the results from Ref. [5] on the study of transferring single-mode squeezing from the microwave field to the magnon mode.

Figures 4(b) and 4(e) show that the magnon squeezing and photon squeezing are quite robust against the cavity damping rate. By noting the magnitude, we can still obtain strong magnon squeezing $(>3 \mathrm{~dB})$ and photon squeezing, even with a low- $\mathcal{Q}$ cavity $\left(\mathcal{Q}=\omega_{a} / \kappa_{a}\right)$. This regime is crucial for detecting the squeezing used in Refs. [64,70], in which the cavity mode has a beam-splitter-like interaction with the magnon mode for reading out the magnon state associated with the CM. In this case $\left(\kappa_{a} \gg \kappa_{m}\right)$, when the drive is switched off and all cavity photons decay, the magnon state remains effectively unchanged, at which time a probe field is sent [4]. Another

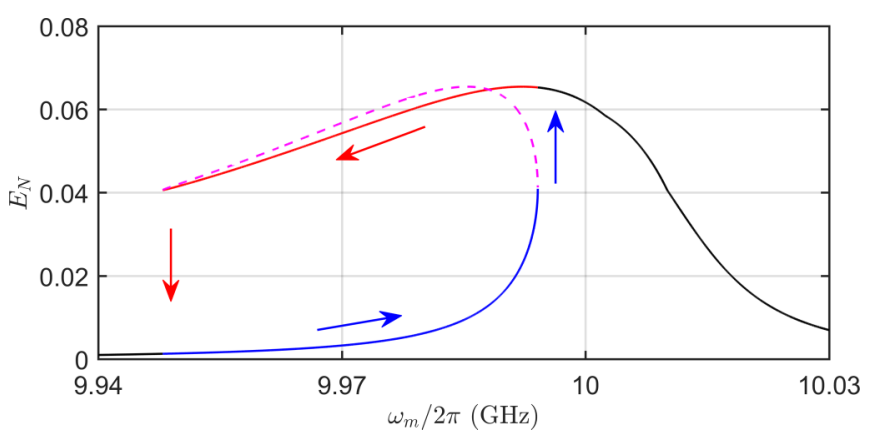

FIG. 5. Magnon-photon entanglement $E_{N}$ versus magnon frequency $\omega_{m}$. The parameters are chosen to be the same as those in Fig. 3(a).

possibility is to study the changes in the squeezing spectrum of the cavity output, which carries a clear signature of magnon squeezing [5,71-73].

The cavity and magnon mode squeezings are also quite robust against the temperature, as shown in Figs. 4(c) and 4(f). Moderate squeezing for both the cavity and magnon mode quadratures can be found even at $T=0.4 \mathrm{~K}$ and $T=0.2 \mathrm{~K}$, respectively. Compared with the use of a single-mode vacuum squeezing field in Ref. [5], the magnon and photon squeezing generated by Kerr nonlinearity are weak. But we can still get a magnon squeezing degree greater than $3 \mathrm{~dB}$. Strong photon squeezing based on state-swap interaction may require magnon-photon ultrastrong coupling [12,74].

\section{MAGNON-PHOTON ENTANGLEMENT}

Besides squeezed states, the system can generate entanglement between photons and magnons. This investigation will use logarithmic negativity [75-77] to quantify the degree of quantum entanglement for the two-mode Gaussian state, which is defined as

$$
E_{N}=\max \left[0,-\ln 2 v^{-}\right],
$$

where $v^{-}=\min$ eig $\left|\oplus_{j=1}^{2}\left(-\sigma_{y}\right) \mathcal{P} \mathcal{V} \mathcal{P}\right|$, with $\sigma_{y}$ and $\mathcal{P}=$ $\sigma_{z} \oplus 1$ being, respectively, the $y$ Pauli matrix and the matrix that realizes partial transposition on the CM [76,78]. Figure 5 illustrates magnon-photon entanglement $E_{N}$ as a function of magnon frequency $\omega_{m}$ with the same parametric mechanism as in Fig. 3(a). There is always a jump from one state to another at two switching points when considering the increasing and decreasing directions of $\omega_{m}$ (i.e., bistable entanglement). A bistable entangled state is essentially derived from the magnon Kerr effect induced by magnetocrystalline anisotropy of the YIG sphere. The entanglement sensitivity at switching points can be considered a response signal, which means that bistable entanglement has particular applications in quantum information processing.

In addition, the entanglement is further demonstrated in Fig. 6 when the CMS does not have a linear relation of $\Delta_{K}=$ $-2 \mathcal{R}$. Since magnon-photon entanglement originates from state-swap interaction and Kerr nonlinearity (quantified by $|\tanh 2 r|)$, there must be an interplay between $g$ and $|\tanh 2 r|$, which is depicted in Fig. 6(a). Figure 6(b) shows considerable entanglement when the system is driven far from equilibrium 

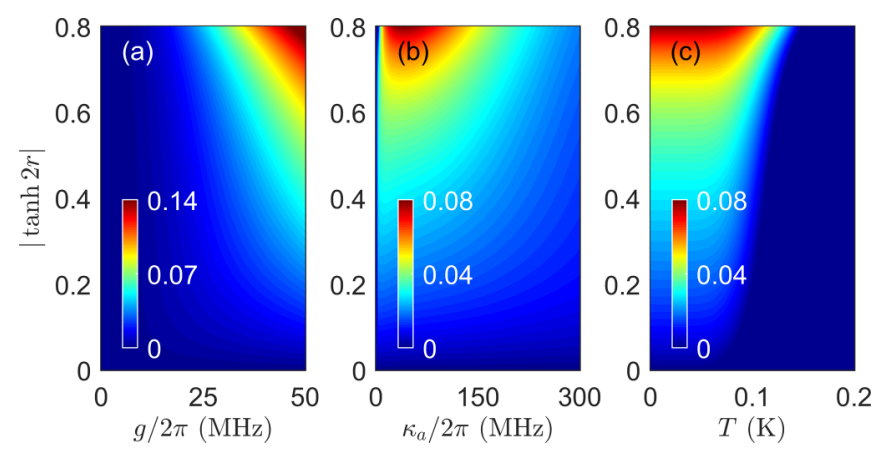

FIG. 6. Three-dimensional plots for magnon-photon entanglement $E_{N}$. We take $\kappa_{a} / 2 \pi=50 \mathrm{MHz}, T=20 \mathrm{mK}$ in (a), $g / 2 \pi=$ $33 \mathrm{MHz}, T=20 \mathrm{mK}$ in (b), and $g / 2 \pi=33 \mathrm{MHz}, \kappa_{a} / 2 \pi=50 \mathrm{MHz}$ in (c), and the other parameters are the same as in Fig. 4.

(i.e., $|\tanh 2 r|>0$ ). This is reasonable because strong external driving significantly enhances Kerr nonlinearity, which is responsible for both squeezing and entanglement (we verify this later on; see Sec. VI). Furthermore, Fig. 6(b) also shows that the larger the cavity damping rate is, the weaker the entanglement observed is. By noting the magnitude, we can still obtain some entanglement, even with a low- $\mathcal{Q}$ cavity showing a magnetically induced transparency regime, where $\kappa_{a}>g>\kappa_{m}$ [34]. In addition, the entanglement is also robust against temperature. We observe moderate magnon-photon entanglement even at $T=0.1 \mathrm{~K}$ [see Fig. 6(c)].

\section{PRINCIPLE AND MECHANISM}

In order to study squeezing and entanglement in more detail, the mechanism for the implementation of the quantum effect by using Kerr nonlinearity should be explained. To this end, we proceed via the effective Hamiltonian for quantum fluctuations

$$
\begin{aligned}
\mathcal{H}_{\mathrm{flu}} / \hbar= & \Delta_{a} \delta a^{\dagger} \delta a+\tilde{\Delta}_{m} \delta m^{\dagger} \delta m+\mathcal{R}\left(\delta m^{\dagger} \delta m^{\dagger}+\delta m \delta m\right) / 2 \\
& +g\left(\delta m^{\dagger} \delta a+\delta m \delta a^{\dagger}\right) .
\end{aligned}
$$

The quadratic terms $\delta m^{\dagger} \delta m^{\dagger}$ and $\delta m \delta m$ imply effective selfinteraction between magnons induced by magnetocrystalline anisotropy, which may be significantly enhanced by strong driving. In addition, the two quadratic terms also the key for creating squeezing and entanglement in nonlinear systems. To show the mechanism, let us diagonalize these two terms by introducing Bogoliubov modes [79,80]: $\delta \beta=$ $\delta m \cosh r+\delta m^{\dagger} \sinh r$, where $|\tanh 2 r|=\left|\mathcal{R} /\left(\Delta_{m}+\Delta_{K}\right)\right|$. Inserting these into Eq. (19), we have the Hamiltonian

$$
\begin{aligned}
\mathcal{H} / \hbar= & \Delta_{a} \delta a^{\dagger} \delta a+\mathcal{B} \delta \beta^{\dagger} \delta \beta+G_{+}\left(\delta \beta^{\dagger} \delta a+\delta \beta \delta a^{\dagger}\right) \\
& +G_{-}\left(\delta \beta^{\dagger} \delta a^{\dagger}+\delta \beta \delta a\right),
\end{aligned}
$$

where $\mathcal{B}=\sqrt{\left(\Delta_{m}+\Delta_{K}\right)^{2}-\mathcal{R}^{2}}, G_{+}=g \cosh r$, and $G_{-}=$ $-g \sinh r$. In general, the magnon mode interacts with a microcavity through beam-splitter-like coupling, which cannot produce any squeezing and entanglement. Nevertheless, activating Kerr nonlinearity via strong driving results in squeezinglike coupling which may let the magnons become squeezed and entangled with cavity photons.
In Hamiltonian (20), $|\tanh 2 r|$ as a squeezing parameter can effectively measure the squeezing degree of magnons. Bistability of the effective Kerr coefficient means the same $\omega_{m}$ can correspond to two different $\Delta_{K}$ (see Fig. 2); that is, there are two different squeezing parameters, which depend on the starting direction of $\omega_{m}$. For example, when $\omega_{m}$ is at the first switching point $\left(\omega_{m} / 2 \pi \simeq 9.95 \mathrm{GHz}\right)$ in Fig. 3(a), the squeezing parameters of the lower stable branch (blue line) and the upper stable branch (red line) $\operatorname{are}|\tanh 2 r| \simeq$ 0 and $|\tanh 2 r| \simeq 0.6$, respectively. This results in different magnon squeezing degrees at the same $\omega_{m}$ in Fig. 3(a). The same physical mechanism can also explain magnon-photon entanglement in Fig. 5. Note that the variation trend of entanglement in Fig. 5 is significantly different from magnon squeezing in Fig. 3(a). Physically, this is easy to understand: the magnon frequency shift induced by the Kerr effect has great influence on entanglement. Therefore, it will directly affect the optimal effective interaction between magnons and photons [4,26]. Our scheme shows the advantage: bistable phenomena in classical physics are introduced into quantum physics, which may serve as an a priori guide for the exploration of bistable behavior of other quantum effects.

\section{PARAMETER FEASIBILITY}

In this section, we briefly discuss the feasibility of the experimental setup that may be used in the experiment. In order to observe rich nonlinear phenomena, we can choose a YIG sphere with a small volume. For example, the Kerr nonlinear coefficient $K / 2 \pi \simeq 1.56 \mu \mathrm{Hz}$ can be achieved in a 40 - $\mu$ m-diameter YIG sphere, while $K / 2 \pi \simeq 10^{-10} \mathrm{~Hz}$ can be gained in a 1-mm-diameter YIG sphere [4]. However, as indicated in Ref. [14], when the size of the sphere is small, the coupling strength between magnons and microwave photons becomes weak. For a fixed single-spin coupling strength $g_{s} / 2 \pi=39 \mathrm{MHz}$, a $40-\mu \mathrm{m}$-diameter (1-mm-diameter) YIG sphere can produce magnon-photon coupling strength $g / 2 \pi \simeq$ 1 (130) MHz. Therefore, a large coupling strength and a small sphere volume seem to be incompatible. However, according to $g_{s}=\sqrt{\gamma^{2} \mu_{0} \hbar \omega_{a} /\left(4 V_{a}\right)}$ and $g=\sqrt{2 S} g_{s}$, we can deduce $g=\sqrt{5 \rho \gamma^{2} \mu_{0} \hbar \omega_{a} V_{m} /\left(4 V_{a}\right)}$. Hence, for a given resonance frequency $\omega_{a}$, the effective coupling is related only to the fill factor $V_{m} / V_{a}$. Therefore, an ordinary 3D copper cavity may not meet the requirement, but a reentrant cavity may satisfy the requirement to enhance $g$. This field-focusing effect of the reentrant cavity results in very high spatial overlap between the cavity mode and the magnon mode, and thus a strong coupling rate between them may be obtained [12].

\section{CONCLUSIONS AND REMARKS}

We have presented a scheme to generate squeezing and entanglement between magnons and microwave photons in a nonlinear CMS and illustrated the bistable behavior of squeezing and entanglement induced by the magnon self-nonlinear Kerr effect by using experimentally feasible parameters. The results show that near the two switching points, the squeezing and entanglement can jump from one state to another state, thus achieving the hysteresis loop phenomenon which is similar to the classic bistable phenomenon. We have also 
shown that by driving the system far from equilibrium, magnons are squeezed in succession. Furthermore, by activating the effective magnon-photon state-swap interaction, the squeezed state is successfully transferred from the magnon mode to the photon mode, and the magnon-photon entanglement is realized.

We should note that our idea for generating bistable quantum states may be potentially extended to other hybrid systems, such as quantum magnonics systems, where a cross-Kerr nonlinearity between the magnetostatic modes and the microwave cavity modes is also made possible by their mutual coupling to qubits. The cross-Kerr nonlinear term is $K_{a c} a^{\dagger} a c^{\dagger} c$, where $K_{a c}$ is the cross-Kerr coefficient, which depends strongly on the magnon frequency relative to the qubit transition frequencies. In the single-excitation-resolved cross-Kerr interaction regime, corresponding to $\left|K_{a c}\right| \gg \kappa_{a}$, $\kappa_{c}$ (the dissipation of modes), a number state in one of the modes can be stabilized through dissipation in the other mode [44]. In particular, these phenomena may be found for molecular aggregates and clusters which own similar forms of nonlinear couplings $b^{\dagger} b q$ and $\Delta b^{\dagger} b b^{\dagger} b$ [7]. With scaled-up parameters, the bistability of quantum states in molecular aggregates would be anticipated in that the exciton-exciton interaction is several orders of magnitude higher than the Kerr nonlinearity resulting from the magnetocrystalline anisotropy. Hence, our scheme may open perspectives for the exploration of the bistable behavior of a quantum state serving in quantum information processing, where the bistability of the quantum state can be used as switches and memories, and may serve as a bridge or transducer, which paves the way for using one effect to induce or control others.

\section{ACKNOWLEDGMENTS}

We thank Prof. A.-D. Zhu for reading the manuscript and providing helpful suggestions. This work is supported by the National Natural Science Foundation of China (Grants No. 11761072 and No. 12001474), the Natural Science Foundation of Fujian Province of China (Grant No. 2018J01661), and the Science and Technology project of the Education Department of Jilin Province of China during the 13th Five-Year Plan Period (Grant No. JJKH20200510KJ).
[1] Z. L. Xiang, S. Ashhab, J. Q. You, and F. Nori, Hybrid quantum circuits: Superconducting circuits interacting with other quantum systems, Rev. Mod. Phys. 85, 623 (2013).

[2] G. Kurizki, P. Bertet, Y. Kubo, K. Mølmer, D. Petrosyan, P. Rabl, and J. Schmiedmayer, Quantum technologies with hybrid systems, Proc. Natl. Acad. Sci. USA 112, 3866 (2015).

[3] J. Li and S. Y. Zhu, Entangling two magnon modes via magnetostrictive interaction, New J. Phys. 21, 085001 (2019).

[4] J. Li, S. Y. Zhu, and G. S. Agarwal, Magnon-Photon-Phonon Entanglement in Cavity Magnomechanics, Phys. Rev. Lett. 121, 203601 (2018).

[5] J. Li, S. Y. Zhu, and G. S. Agarwal, Squeezed states of magnons and phonons in cavity magnomechanics, Phys. Rev. A 99, 021801(R) (2019).

[6] C. Kong, H. Xiong, and Y. Wu, Magnon-Induced Nonreciprocity Based on the Magnon Kerr Effect, Phys. Rev. Appl. 12, 034001 (2019).

[7] Z. Zhang, M. O. Scully, and G. S. Agarwal, Quantum entanglement between two magnon modes via Kerr nonlinearity driven far from equilibrium, Phys. Rev. Res. 1, 023021 (2019).

[8] H. Y. Yuan and X. R. Wang, Magnon-photon coupling in antiferromagnets, Appl. Phys. Lett. 110, 082403 (2017).

[9] H. Y. Yuan, S. Zhang, Z. Ficek, Q. Y. He, and M. H. Yung, Enhancement of magnon-magnon entanglement inside a cavity, Phys. Rev. B 101, 014419 (2020).

[10] S. S. Zheng, F. X. Sun, H. Y. Yuan, Z. Ficek, Q. H. Gong, and Q. Y. He, Enhanced entanglement and asymmetric EPR steering between magnons, Sci. China: Phys., Mech. Astron. 64, 210311 (2020).

[11] H. Y. Yuan, P. Yan, S. S. Zheng, Q. Y. He, K. Xia, and M. H. Yung, Steady Bell State Generation via Magnon-Photon Coupling, Phys. Rev. Lett. 124, 053602 (2020).

[12] M. Goryachev, W. G. Farr, D. L. Creedon, Y. Fan, M. Kostylev, and M. E. Tobar, High-Cooperativity Cavity QED with Magnons at Microwave Frequencies, Phys. Rev. Appl. 2, 054002 (2014).
[13] Z. B. Yang, X. D. Liu, X. Y. Yin, Y. Ming, H. Y. Liu, and R. C. Yang, Controlling Stationary One-Way Quantum Steering in Cavity Magnonics, Phys. Rev. Appl. 15, 024042 (2021).

[14] G. Q. Zhang, Y. P. Wang, and J. Q. You, Theory of the magnon Kerr effect in cavity magnonics, Sci. China: Phys., Mech. Astron. 62, 987511 (2019).

[15] Ö. O. Soykal and M. E. Flatté, Strong Field Interactions between a Nanomagnet and a Photonic Cavity, Phys. Rev. Lett. 104, 077202 (2010).

[16] Ö. O. Soykal and M. E. Flatté, Size dependence of strong coupling between nanomagnets and photonic cavities, Phys. Rev. B 82, 104413 (2010).

[17] B. Z. Rameshti, Y. Cao, and G. E. W. Bauer, Magnetic spheres in microwave cavities, Phys. Rev. B 91, 214430 (2015).

[18] H. Huebl, C. W. Zollitsch, J. Lotze, F. Hocke, M. Greifenstein, A. Marx, R. Gross, and S. T. B. Goennenwein, High Cooperativity in Coupled Microwave Resonator Ferrimagnetic Insulator Hybrids, Phys. Rev. Lett. 111, 127003 (2013).

[19] Y. Tabuchi, S. Ishino, T. Ishikawa, R. Yamazaki, K. Usami, and Y. Nakamura, Hybridizing Ferromagnetic Magnons and Microwave Photons in the Quantum Limit, Phys. Rev. Lett. 113, 083603 (2014).

[20] X. Zhang, C. L. Zou, L. Jiang, and H. X. Tang, Strongly Coupled Magnons and Cavity Microwave Photons, Phys. Rev. Lett. 113, 156401 (2014).

[21] D. Zhang, X. M. Wang, T. F. Li, X. Q. Luo, W. Wu, F. Nori, and J. Q. You, Cavity quantum electrodynamics with ferromagnetic magnons in a small yttrium-iron-garnet sphere, npj Quantum Inf. 1, 15014 (2015).

[22] Y. P. Wang, J. W. Rao, Y. Yang, P. C. Xu, Y. S. Gui, B. M. Yao, J. Q. You, and C. M. Hu, Nonreciprocity and Unidirectional Invisibility in Cavity Magnonics, Phys. Rev. Lett. 123, 127202 (2019).

[23] C. Kittel, On the theory of ferromagnetic resonance absorption, Phys. Rev. 73, 155 (1948). 
[24] C. Kong, B. Wang, Z. X. Liu, H. Xiong, and Y. Wu, Magnetically controllable slow light based on magnetostrictive forces, Opt. Express 27, 5544 (2019).

[25] L. Bai, M. Harder, Y. P. Chen, X. Fan, J. Q. Xiao, and C. M. Hu, Spin Pumping in Electrodynamically Coupled Magnon-Photon Systems, Phys. Rev. Lett. 114, 227201 (2015).

[26] Y. P. Wang, G. Q. Zhang, D. Zhang, T. F. Li, C. M. Hu, and J. Q. You, Bistability of Cavity Magnon Polaritons, Phys. Rev. Lett. 120, 057202 (2018).

[27] Y. P. Wang, G. Q. Zhang, D. Zhang, X. Q. Luo, W. Xiong, S. P. Wang, T. F. Li, C. M. Hu, and J. Q. You, Magnon Kerr effect in a strongly coupled cavity-magnon system, Phys. Rev. B 94, 224410 (2016).

[28] Z. X. Liu, B. Wang, H. Xiong, and Y. Wu, Magnoninduced high-order sideband generation, Opt. Lett. 43, 3698 (2018).

[29] B. Wang, Z. X. Liu, C. Kong, H. Xiong, and Y. Wu, Magnon induced transparency and amplification in PT-symmetric cavity magnon system, Opt. Express 26, 20248 (2018).

[30] R. Hisatomi, A. Osada, Y. Tabuchi, T. Ishikawa, A. Noguchi, R. Yamazaki, K. Usami, and Y. Nakamura, Bidirectional conversion between microwave and light via ferromagnetic magnons, Phys. Rev. B 93, 174427 (2016).

[31] X. Zhang, C. L. Zou, N. Zhu, F. Marquardt, L. Jiang, and H. X. Tang, Magnon dark modes and gradient memory, Nat. Commun. 6, 8914 (2015).

[32] B. Yao, Y. S. Gui, J. W. Rao, S. Kaur, X. S. Chen, W. Lu, Y. Xiao, H. Guo, K. P. Marzlin, and C. M. Hu, Cooperative polariton dynamics in feedback-coupled cavities, Nat. Commun. 8, 1437 (2017).

[33] M. Harder, L. Bai, P. Hyde, and C. M. Hu, Topological properties of a coupled spin-photon system induced by damping, Phys. Rev. B 95, 214411 (2017).

[34] D. Zhang, X. Q. Luo, Y. P. Wang, T. F. Li, and J. Q. You, Observation of the exceptional point in cavity magnonpolaritons, Nat. Commun. 8, 1368 (2017).

[35] X. Zhang, C. L. Zou, L. Jiang, and H. X. Tang, Cavity magnomechanics, Sci. Adv. 2, e1501286 (2016).

[36] Y. Tabuchi, S. Ishino, A. Noguchi, T. Ishikawa, R. Yamazaki, K. Usami, and Y. Nakamura, Coherent coupling between a ferromagnetic magnon and a superconducting qubit, Science 349, 405 (2015).

[37] D. Lachance-Quirion, Y. Tabuchi, S. Ishino, A. Noguchi, T. Ishikawa, R. Yamazaki, and Y. Nakamura, Resolving quanta of collective spin excitations in a millimeter-sized ferromagnet, Sci. Adv. 3, e1603150 (2017).

[38] J. A. Haigh, S. Langenfeld, N. J. Lambert, J. J. Baumberg, A. J. Ramsay, A. Nunnenkamp, and A. J. Ferguson, Magneto-optical coupling in whispering-gallery-mode resonators, Phys. Rev. A 92, 063845 (2015).

[39] A. Osada, R. Hisatomi, A. Noguchi, Y. Tabuchi, R. Yamazaki, K. Usami, M. Sadgrove, R. Yalla, M. Nomura, and Y. Nakamura, Cavity Optomagnonics with Spin-Orbit Coupled Photons, Phys. Rev. Lett. 116, 223601 (2016).

[40] X. Zhang, N. Zhu, C. L. Zou, and H. X. Tang, Optomagnonic Whispering Gallery Microresonators, Phys. Rev. Lett. 117, 123605 (2016).

[41] J. A. Haigh, A. Nunnenkamp, A. J. Ramsay, and A. J. Ferguson, Triple-Resonant Brillouin Light Scattering in Magneto-Optical Cavities, Phys. Rev. Lett. 117, 133602 (2016).
[42] S. Sharma, Y. M. Blanter, and G. E. W. Bauer, Optical Cooling of Magnons, Phys. Rev. Lett. 121, 087205 (2018).

[43] A. Osada, A. Gloppe, R. Hisatomi, A. Noguchi, R. Yamazaki, M. Nomura, Y. Nakamura, and K. Usami, Brillouin Light Scattering by Magnetic Quasivortices in Cavity Optomagnonics, Phys. Rev. Lett. 120, 133602 (2018).

[44] D. Lachance-Quirion, Y. Tabuchi, A. Gloppe, K. Usami, and Y. Nakamura, Hybrid quantum systems based on magnonics, Appl. Phys. Exp. 12, 070101 (2019).

[45] S. V. Kusminskiy, Cavity optomagnonics, Optomagnonic Structures (World Scientific, Singapore, 2021), pp. 299-353, Chap. 7.

[46] D. D. Stancil and A. Prabhakar, Spin Waves (Springer, Berlin, 2009).

[47] A. G. Gurevich and G. A. Melkov, Magnetization Oscillations and Waves (CRC Press, Boca Raton, FL, 1996).

[48] S. R. K. Rodriguez, W. Casteels, F. Storme, N. Carlon Zambon, I. Sagnes, L. L. Gratiet, E. Galopin, A. Lemaître, A. Amo, C. Ciuti, and J. Bloch, Probing a Dissipative Phase Transition via Dynamical Optical Hysteresis, Phys. Rev. Lett. 118, 247402 (2017).

[49] F. Letscher, O. Thomas, T. Niederprüm, M. Fleischhauer, and H. Ott, Bistability Versus Metastability in Driven Dissipative Rydberg Gases, Phys. Rev. X 7, 021020 (2017).

[50] T. K. Paraïso, M. Wouters, Y. Leéger, F. Morier-Genoud, and B. Deveaud-Plédran, Multistability of a coherent spin ensemble in a semiconductor microcavity, Nat. Mater. 9, 655 (2010).

[51] O. R. Bilal, A. Foehr, and C. Daraio, Bistable metamaterial for switching and cascading elastic vibrations, Proc. Natl. Acad. Sci. U.S.A. 114, 4603 (2017).

[52] E. Kuramochi, K. Nozaki, A. Shinya, K. Takeda, T. Sato, S. Matsuo, H. Taniyama, H. Sumikura, and M. Notomi, Large-scale integration of wavelength-addressable all-optical memories on a photonic crystal chip, Nat. Photonics 8, 474 (2014).

[53] V. Kubytskyi, S. A. Biehs, and P. Ben-Abdallah, Radiative Bistability and Thermal Memory, Phys. Rev. Lett. 113, 074301 (2014).

[54] C. M. Caves, K. S. Thorne, R. W. P. Drever, V. D. Sandberg, and M. Zimmermann, On the measurement of a weak classical force coupled to a quantum-mechanical oscillator. I. Issues of principle, Rev. Mod. Phys. 52, 341 (1980).

[55] A. Bassi, K. Lochan, S. Satin, T. P. Singh, and H. Ulbricht, Models of wave-function collapse, underlying theories, and experimental tests, Rev. Mod. Phys. 85, 471 (2013).

[56] S. L. Braunstein and P. van Loock, Quantum information with continuous variables, Rev. Mod. Phys. 77, 513 (2005).

[57] T. Holstein and H. Primakoff, Field Dependence of the Intrinsic Domain Magnetization of a Ferromagnet, Phys. Rev. 58, 1098 (1940).

[58] A. F. Kockum, A. Miranowicz, S. D. Liberato, S. Savasta, and F. Nori, Ultrastrong coupling between light and matter, Nat. Rev. Phys. 1, 19 (2019).

[59] S. Haroche and J. M. Raymond, Exploring the Quantum: Atoms, Cavities, and Photons (Oxford University Press, Oxford, 2006).

[60] X. Gu, A. F. Kockum, A. Miranowicz, Y.-X. Liu, and F. Nori, Microwave photonics with superconducting quantum circuits, Phys. Rep. 718-719, 1 (2017).

[61] C. W. Gardiner and P. Zoller, Quantum Noise (Springer, New York, 2004). 
[62] Here, we adopt a slightly smaller number of parameters that are different from those of Ref. [26] in the study of squeezing and entanglement. One may choose the parameters according to the purpose of producing bistable quantum states.

[63] E. X. DeJesus and C. Kaufman, Routh-Hurwitz criterion in the examination of eigenvalues of a system of nonlinear ordinary differential equations, Phys. Rev. A 35, 5288 (1987).

[64] D. Vitali, S. Gigan, A. Ferreira, H. R. Böhm, P. Tombesi, A. Guerreiro, V. Vedral, A. Zeilinger, and M. Aspelmeyer, Optomechanical Entanglement between a Movable Mirror and a Cavity Field, Phys. Rev. Lett. 98, 030405 (2007).

[65] S. Chakraborty and A. K. Sarma, Entanglement dynamics of two coupled mechanical oscillators in modulated optomechanics, Phys. Rev. A 97, 022336 (2018).

[66] W. Vogel and D. G. Welsch, Quantum Optics (Wiley-VCH, Weinheim, 2006)

[67] C. Weedbrook, S. Pirandola, R. García-Patrón, N. J. Cerf, T. C. Ralph, J. H. Shapiro, and S. Lloyd, Gaussian quantum information, Rev. Mod. Phys. 84, 621 (2012).

[68] Note that a large value of $\Delta_{K}$ is selected in Fig. 4 in order to simulate the general situation of magnon squeezing. $\Delta_{K} / 2 \pi=$ $280 \mathrm{MHz}$ corresponds to $\left\langle m^{\dagger} m\right\rangle \simeq 4.48 \times 10^{13} \ll 2 S$ [4]. In this case, the low-lying excitation approximation mentioned earlier is still valid.

[69] In Fig. 4(a), with the increase of $g$, the squeezing degree of the magnon mode has a tendency to increase, which may be related to the cavity-cooling effect.
[70] T. A. Palomaki, J. D. Teufel, R. W. Simmonds, and K. W. Lehnert, Entangling mechanical motion with microwave fields, Science 342, 710 (2013).

[71] P. C. Parks and V. Hahn, Stability Theory (Prentice Hall, New York, 1993).

[72] M. J. Collett and C. W. Gardiner, Squeezing of intracavity and traveling-wave light fields produced in parametric amplification, Phys. Rev. A 30, 1386 (1984).

[73] S. Huang and G. S. Agarwal, Entangling nanomechanical oscillators in a ring cavity by feeding squeezed light, New J. Phys. 11, 103044 (2009).

[74] J. Bourhill, N. Kostylev, M. Goryachev, D. L. Creedon, and M. E. Tobar, Ultrahigh cooperativity interactions between magnons and resonant photons in a YIG sphere, Phys. Rev. B 93, 144420 (2016).

[75] J. Eisert, Ph.D. thesis, Entanglement in Quantum Information Theory, University of Potsdam, 2001.

[76] G. Vidal and R. F. Werner, Computable measure of entanglement, Phys. Rev. A 65, 032314 (2002).

[77] M. B. Plenio, Logarithmic Negativity: A Full Entanglement Monotone That Is Not Convex, Phys. Rev. Lett. 95, 090503 (2005).

[78] R. Simon, Peres-Horodecki Separability Criterion for Continuous Variable Systems, Phys. Rev. Lett. 84, 2726 (2000).

[79] E. M. Lifshitz and L. P. Pitaevskii, Statistical Physics: Theory of the Condensate State, Part 2, rev. ed. (Butterworth-Heinemann, Oxford, 1980).

[80] A. L. Fetter and J. D. Walecka, Quantum Theory of ManyParticle Systems (Dover, Mineola, NY, 2003). 